Interactive comment on "Mass-conserving coupling of total column $\mathrm{CO}_{2}\left(\mathrm{XCO}_{2}\right)$ from global to mesoscale models: Case study with CMS-Flux inversion system and WRF-Chem (v3.6.1)" by Martha P. Butler et al.

Martha P. Butler et al.

sfeng@psu.edu

Received and published: 31 October 2019

Response to Anonymous Referee \# 2

"Mass-conserving coupling of total column CO2Âă(XCO2) from global to mesoscale models: Case study with CMS-Flux inversion system and WRF-Chem (v3.6.1)" [gmd-2018-342], Butler et al. 


\section{General Remarks:}

We greatly appreciate the thoughtful and constructive suggestions from Anonymous Referee \# 2. We have addressed all of the comments and made the revised manuscript clearer. Point-by-point responses follow. The original comments from the reviewer are in italics and the response in normal.

\section{General remarks}

The authors compare total column CO2 over North America computed from WRFChem with results from the CMS-Flux inversion system which is based on GEOSChem. For this purpose they developed a scheme for nesting WRF-Chem into GEOSChem in a way that the mass of $\mathrm{CO} 2$ introduced into WRF-Chem from GEOS-Chem is conserved. Although only minor differences between the results of the two models were found for the total vertical column of $\mathrm{CO} 2$, more pronounced differences were found between the vertical CO2 distributions computed by the two models.

This investigation could generally be useful for the community who do inversions of satellite derived $\mathrm{CO} 2$ concentrations. However, the paper suffers in major parts from imprecise language and unclear descriptions of important aspects. In particular, the description of the methods does not allow finding out whether the very little added value of the higher spatial resolution could eventually be attributed to the way how surface CO2 fluxes are implemented in WRF-Chem. I cannot recommend the publication of this paper unless the paper is improved in these aspects.

Printer-friendly version

Response: We thank the reviewers for the valuable comments. We have clarified the implementation of CMS fluxes into WRF. We have used no interpolation nor projection

Discussion paper 
tools but instead directly assigned CMS low-resolution fluxes to the high-resolution WRF pixels within the grid box. Scaling factors were applied only to compensate for coastal areas and small changes in total fluxes due to the pixel attribution between WRF and CMS. These adjustments remain small compared to the actual fluxes and fully preserve the flux distribution. We have clarified these different points in the main text.

\section{Detailed comments}

Throughout the paper the language of the paper is imprecise which makes major parts of the paper hard to read. Already the abstract is a good example:

What is meant by 'fluxes' in the first sentence? Fluxes from the surface? Fluxes from which sources?

We have clarified that point. "CO2 surface fluxes"

What is meant by 'transport'? Long range transport?

We have clarified that point. "atmospheric transport simulations"

What is 'our' North American domain?

We have clarified that point.

A few further examples (by far not complete) are:

Page 1, last line: 'do not agree well': With what?

Printer-friendly version

Page 2, lines 21 and 23: The expression 'curtains' is somewhat odd.

Discussion paper 
Response: We have clarified the text as suggested. Please find the revised version with colored modifications.

Page 6, first line: What does 'dried of water vapor' mean here (odd wording anyway)?

Response: The GEOS-Chem model had an incorrect representation of mole fractions which included, in part, water vapor in the total mass of air. We had to remove the contribution of water vapor to the total air mass before coupling to the WRF model. We have clarified in the text: "after correcting for the presence of water vapor to obtain dry air mole fractions" .

The meteorological driver of GEOS-Chem is GEOS-5 while meteorological boundary conditions for WRF-Chem are from ERA interim. In how far can differences between these meteorological drivers contribute to the differences in upper air wind fields and CO2 concentrations from WRF-Chem and GEOS-Chem. This should be analyzed in more detail.

Response: We agree with the reviewer that we have coupled the mole fractions from $\mathrm{CMS}$ and not the actual mass fluxes of $\mathrm{CO} 2$ molecules through the boundaries. Therefore, driver data will play a significant role between the two models at daily to weekly time scales. While we acknowledge the fact that wind fields will vary between the two models, this question would lead to separating the impact of the WRF model physics from the driver data. This comparison would be worthwhile but we do not expect significant variations at seasonal time scales. Both systems (GEOS and ERA-I) assimilate similar meteorological observations, hence represent the mean seasonal wind in similar ways. We have investigated the impact of driver data in regional simulations of CO2 (Diaz-Isaac et al., 2018) and found that daily variations from different driver data

Printer-friendly version

Discussion paper 
can be large, but we do not expect major differences at the seasonal scale. We also note that GEOS-Chem has its own model schemes to utilize the wind from GEOS-5 to transport $\mathrm{CO} 2$, which differs from WRF (advection, diffusion, convection schemes). This additional layer will confuse the comparison whether differences come from driver data or from the model physics. We have added text in the manuscript. "The two different re-analysis driver data used here might also cause differences in simulated $\mathrm{XCO} 2$ and $\mathrm{CO} 2$ mole fractions. No reconciliation was performed because both models re-interpret driver data to a certain extent (through advection and diffusion schemes). However, comparison of ERA-Interim and GEOS-5 driver data would potentially bring additional information about transport differences."

The authors describe in much detail how they achieve mass conservation when deriving CO2 surface fluxes for the WRF-Chem simulation from the fluxes applied to GEOS-Chem. Does this result in surface fluxes smoothed to the GEOS-Chem grid, which are used as input for WRF-Chem? What CO2 emission patterns (anthropogenic, biogenic) are still resolved in the WRF-Chem simulation? It is not clear whether the emissions for the WRF-Chem simulations are really better spatially resolved than for the GEOS-Chem simulations. Eventually show emission input for WRF-Chem and for GEOS-Chem in the supplementary material.

Response: Mass-conservation for the fluxes is due to mis-alignment of coastal fluxes, near water bodies, and to small mismatches when assigning a small WRF pixel to the corresponding large CMS pixel. No smoothing nor any deformation was applied when re-gridding the surface fluxes. The scaling factor is computed over the entire domain and applied to the entire domain. The spatial distribution of the fluxes remains identical in WRF and CMS. And all flux components from the CMS model are used to have identical surface fluxes in both models. We have clarified these points in the text (Method section).

Printer-friendly version

Discussion paper 
Response: To clarify, the same diurnal cycle of the fluxes is applied to both models. Only the scaling factor (correction after re-gridding) of the fluxes is kept constant over a month, instead of being calculated for each hourly flux map. The result is minor (few tenths of a percent) compared to scaling for all the 3-hourly fluxes. We have clarified the text.

Section 2.4.2: Why is the averaging performed over such a big area?

Response: The sparsity of GOSAT soundings forces us to select a wide area around the TCCON site. Ideally, this box would be limited to a few degrees, or based on synoptic-scale pressure fields. To have a sufficient number of GOSAT soundings, we extended the box to $6 \times 12$ degree in size. Other methods have been developed (Guerlet et al., 2013) but rely on simulated CO2 fields which vary depending on the model used. We clarified in the text.

Page 9, lines 3-6: These sentences are hard to understand. This should be explained in more detail. Please mention also the magnitude of the differences between the individual GOSAT XCO2 soundings which are located within a single GEOS-Chem cell?

Response: We have clarified the text. The sampling density of GOSAT remains very low for any given CMS grid cell. The number of soundings in a typical CMS grid cell varies greatly with an annual estimate, between 1 to 300 soundings per grid cell. Over Summer, we applied a threshold of 10 soundings per grid cell, or one every three days.

Printer-friendly version

Discussion paper 
In other terms, differences within a grid cell would quantify day-to-day variations but rarely overlaps in the same day.

Page 12, line 14: Why is convective transport of CO2 not included? WRF-Chem can handle convective vertical transport of atmospheric trace compounds.

Response: We have explored in great details the problem of convection parameterization in WRF-Chem. Currently, the WRF-Chem code includes an offline parameterization of convective tracer transport based on precipitation rates. As we have carefully investigated, we have found no direct coupling between mass fluxes within the convection scheme and the chemistry code. We are currently working on that issue and will publish results in the coming months. We agree with the reviewer's comment that we could have used the offline coupling (conv_tr). In this simulation, we have turned on the convection scheme but no direct coupling to the chemistry scheme was used. We hope to fix that problem in future simulations.

The conclusions must be extended. Currently they contain mostly a description of data availability and a short summary

Response: We have modified significantly the conclusions, focusing on a description of our results which includes the comparison of modeled mixing ratios to satellite, aircraft and tower observations. 